\title{
1 Rostral Anterior Cingulate Activations inversely relate to Reward Payoff Maximation \& predict Depressed Mood
}

3 Pragathi Priyadharsini Balasubramani ${ }^{1 *}$, Juan Diaz-Delgado ${ }^{1}$, Gillian Grennan $^{1}$, Fahad Alim $^{1}$, 4 Mariam Zafar-Khan ${ }^{1}$, Vojislav Maric ${ }^{1}$, Dhakshin Ramanathan ${ }^{1,2}$, Jyoti Mishra ${ }^{\text {* }}$

$5 \quad{ }^{1}$ Neural Engineering and Translation Labs, Department of Psychiatry, University of California, San Diego, La Jolla, CA, USA

${ }^{2}$ Department of Mental Health, VA San Diego Medical Center, San Diego, CA

8

9

10 *Correspondence should be addressed to:

11 Pragathi Priyadharsini Balasubramani \& Jyoti Mishra

12 University of California, San Diego

13 Neural Engineering \& Translation Labs (NEAT Labs)

149500 Gilman Drive Mail Code 0875

$15 \quad$ La Jolla, CA 92037

16 pbalasubramani@health.ucsd.edu \& jymishra@health.ucsd.edu

19 Keywords: reward prediction; risk sensitivity; reinforcement learning; anterior cingulate cortex;;

20 depression

Conflict of Interest. The authors declare no conflict of interest. 


\section{Abstract}

Choice selection strategies and decision making are typically investigated using multiple-

28 choice gambling paradigms that require participants to maximize reward payoff. However,

29 research shows that performance in such paradigms suffers from individual biases towards the

30 frequency of gains to choose smaller local gains over larger longer term gain, also referred to as

31 melioration. Here, we developed a simple two-choice reward task, implemented in 186 healthy

32 human adult subjects across the adult lifespan to understand the behavioral, computational, and

33 neural bases of payoff maximization versus melioration. The observed reward choice behavior on

34 this task was best explained by a reinforcement learning model of differential future reward

35 prediction. Simultaneously recorded and source-localized electroencephalography (EEG) showed

36 that diminished theta-band activations in the right rostral anterior cingulate cortex (rACC)

37 correspond to greater reward payoff maximization, specifically during the presentation of

38 cumulative reward information at the end of each task trial. Notably, these activations (greater

39 rACC theta) predicted depressed mood symptoms, thereby showcasing a reward processing 40 marker of potential clinical utility. 


\section{Significance Statement}

This study presents cognitive, computational and neural (EEG-based) analyses of a rapid

44 reward-based decision-making task. The research has the following three highlights. 1) It teases

45 apart two core aspects of reward processing, i.e. long term expected value maximization versus

46 immediate gain frequency melioration based choice behavior. 2) It models reinforcement learning

47 based behavioral differences between individuals showing that observed performance is best

48 explained by differential extents of reward prediction. 3) It investigates neural correlates in 186

49 healthy human subjects across the adult lifespan, revealing specific theta band cortical source

50 activations in right rostral anterior cingulate as correlates for maximization that further predict

51 depressed mood across subjects.

\section{Introduction}

Cognitive and neural responses to reward and risk are quintessential to understanding

55 human behavior. Gambling tasks predominantly form the experimental test beds for measuring

56 reward and risk processing abilities in humans (Bechara et al., 1997; Brevers et al., 2013).

57 However, it is widely debated how well these tasks can separate decision-making based on

58 frequency of gains/losses versus expected value of payoff for different choice-sets (Bechara et al.,

59 1997, 2000, 2000; Bembich et al., 2014; Christakou et al., 2009; Garon et al., 2006; R. Gupta et

60 al., 2011; Mueller et al., 2010; Must et al., 2013; Oberg et al., 2011; Roca et al., 2008; Woodrow

61 et al., 2019).

For instance, studies that have controlled for gain frequency in the Iowa Gambling Task

63 (IGT) show that subject choices reflect their inherent gain frequency preferences in the task, which 
64 portray relatively immediate reinforcement based choice behavior rather than a behavior that 65 maximizes expected value or long-term payoff (Chiu et al., 2008; Chiu \& Lin, 2007; Lin et al., 66 2009; Napoli \& Fum, 2010; Singh \& Khan, 2012). In contrast, some researchers suggest such local 67 minima choices also referred to as melioration may be the rational solution in the face of 68 uncertainty (Sims et al., 2013). Additionally, task related performance measures for long-term 69 payoff versus immediate gain frequency bias may reflect distinct reinforcement learning and 70 decision making strategies, e.g. sensitivity to rewards and risks, learning rate, and other behavioral 71 execution strategies (Franken \& Muris, 2005; Furl, 2010; Harman, 2011; Newman et al., 2008;

72 Weller et al., 2010), which prior neural studies of reward payoff maximization have not accounted 73 for.

74 In this study, we uniquely separate immediate gain frequency bias driven decision-making 75 from advantageous longer term payoff-based decision making. Specifically, we designed a two-

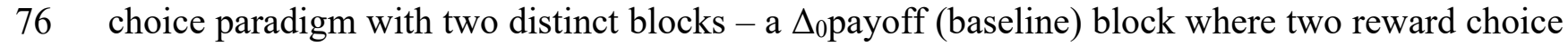
77 options have equal payoffs and reward variance suitable for measuring the immediate gain 78 frequency bias, and a $\Delta$ payoff (difference) block where the two-choice options have unequal 79 payoffs suitable for measuring payoff influences. We thereby, tease apart measurements of 80 immediate gain frequency biased response from expected value or long-term payoff based 81 response, to understand the distinct cognitive and neural mechanisms underlying payoff decisions. Second, we capitalize on computational reinforcement learning (RL) models to understand 83 the basis of individual differences in reward and risk based learning across subjects 84 (Balasubramani et al., 2014; Balasubramani \& Chakravarthy, 2019; Chakravarthy et al., 2018 ; A.

85 Gupta et al., 2013; Muralidharan et al., 2014). The RL framework provides the ability to simulate 86 the observed behavior and estimate the hidden parameters forming the basis for individual 
87 differences in learning and performance. Specifically in this study, we checked whether the

88 observed subject behavior for reward payoff maximization versus gain frequency bias melioration

89 can be explained by extent of future reward prediction (Doya, 2002), or differential risk seeking

90 towards gains versus losses (Balasubramani et al., 2014).

91 Neurally, earlier studies have suggested the significant role of frontal executive regions,

92 particularly the medial prefrontal cortex (mPFC) in reward and risk based performance

93 (Balasubramani \& Hayden, 2018; Bechara et al., 1997, 2000; Brevers et al., 2013; Kennerley et

94 al., 2011; Moccia et al., 2017). Uniquely, in this study, we estimate the neural correlates for payoff

95 relevant decisions while accounting for significant RL parameters and individual differences in

96 immediate gain frequency biases. Finally, we understand the relationship between the identified

97 neural correlates for reward maximization to individual's self-reported mood symptom variations.

98 We show that reward payoff maximization correlates within $\mathrm{mPFC}$ are sensitive to depressed

99 mood, and hence our experimental and computational framework for identifying the regions of 100 interest in mPFC may serve future clinical utility as a biomarker for depression. 


\section{Methods}

103

104

105

106

107

108

109

110

111

112

113

114

115

116

117

118

119

120

121

122

123

124

125

126

127

128

129

130

131

132

133

134

135

136

137

138

139

140

141

142

143

144

145

146

147

Participants. 198 adult human subjects (age mean \pm standard deviation $35.44 \pm 20.30$ years, range 18-80 years, 115 females) participated in the study. All participants provided written informed consent for the study protocol approved by the University of California San Diego institutional review board (UCSD IRB \#180140). Twelve of these participants were excluded from the study as they had a current diagnosis for a psychiatric disorder and current/recent history of psychotropic medications for a final sample of 186 healthy adult participants. All participants reported normal/corrected-to-normal vision and hearing and no participant reported color blindness. For older adults $>60$ years of age, participants were confirmed to have a Mini-Mental State Examination (MMSE) score $>26$ to verify absence of apparent cognitive impairment (ArevaloRodriguez et al. 2015). All data was collected prior to the COVID-19 period of restricted human subjects research.

Surveys. All participants provided demographic information by self-report including age, gender, race (in a scale of 1 to 7: Caucasian; Black/African American; Native Hawaiian / Other Pacific Islander; Asian; American Indian / Alaska Native; More than one race; Unknown or not reported) and ethnicity; socio-economic status (SES) was measured on the Family Affluence Scale from 1 to 9 (Boudreau and Poulin, 2008), and any current/past history of clinical diagnoses and medications were reported. For older adults $>60$ years of age, participants completed the MiniMental State Examination (MMSE) and scored $>26$ to verify absence of apparent cognitive impairment (Arevalo-Rodriguez et al., 2015). All participants completed subjective mental health self-reports using standard instruments, ratings of inattention and hyperactivity obtained on the Adult ADHD Rating Scale (New York University and Massachusetts General Hospital. Adult ADHD-RS-IV with Adult Prompts. 2003; : 9-10), Generalized Anxiety Disorder 7-item scale GAD-7 (Spitzer et al., 2006) and depression symptoms reported on the 9-item Patient Health Questionnaire, PHQ-9 (Kroenke et al., 2001). Symptoms for these psychiatric conditions were measured because they have been related to changes in reward processing (Admon \& Pizzagalli, 2015; Dillon et al., 2014; Luman et al., 2010).

Task Design. We investigated a two-choice decision-making task that enabled a rapid assessment and was easy to understand across the adult lifespan. In this task that we refer to as Lucky Door, participants chose between one of two doors, either a rare gain door (RareG, probability for gains $\mathrm{p}=0.3$, for losses $\mathrm{p}=0.7$ ) or a rare loss door (RareL, probability for losses $\mathrm{p}=0.3$, for gains $\mathrm{p}=0.7$ ). Participants used the left and right arrow keys on the keyboard to make their door choice. Door choice was monitored throughout the task. In two separate blocks, we investigated whether the overall expected value (payoff) of the choice door can influence individual behavior. In the baseline block with $\Delta_{0}$ payoff (no-payoff difference), the two choice doors did not differ in payoff (RareL door, $\mathrm{p}=0.3$ for -70 coins and $\mathrm{p}=0.7$ for +30 coins, payoff $=0$; RareG door, $\mathrm{p}=0.3$ for +70 coins and $\mathrm{p}=0.7$ for -30 coins, payoff $=0$ ). In the experimental difference block with $\Delta$ payoff (payoff difference), expected value or payoff was greater for the RareG door ( $\mathrm{p}=0.3$ for +60 coins, $p=0.7$ for -20 coins, payoff $=+40)$ than for the RareL door $(p=0.3$ for -60 coins, $p=0.7$ for +20 coins; payoff $=-40$ ). Manipulation of payoff, with greater expected value tied to the RareG door, allowed for investigating individual propensities to prioritize long-term (or cumulative) vs. short-term (or immediate) rewards. The RareG door was assigned greater payoff because selecting this door suggests payoff-based decision processing in subjects as opposed to simply choosing based on 
frequency of gains, in which case the RareL choice should be preferred. 40 trials were presented per block and block order was randomized across participants; two practice trials preceded the main $\Delta$ payoff or $\Delta_{0}$ payoff blocks. Figure 1A shows a schematic of the task stimulus sequence and Supplementary table 1 shows the reward distribution that was shuffled and updated after every 10 trials had been sampled from that set.

The Lucky Door task was deployed in Unity as part of the assessment suite on the BrainE (short for Brain Engagement) platform (Balasubramani et al., 2020). The Lab Streaming Layer (LSL (Kothe et al., 2019)) protocol was used to time-stamp each stimulus/response event during the task. Study participants engaged with the assessment on a Windows 10 laptop sitting at a comfortable viewing distance.

Electroencephalography (EEG). EEG data was collected simultaneous to the Lucky Door task using a 24-channel Smarting device with a semi-dry and wireless electrode layout (Next EEGnew human interface, MBT). Data were acquired at $500 \mathrm{~Hz}$ sampling frequency at 24-bit resolution. Cognitive event markers were integrated using LSL and data files were stored in xdf format.

Behavioral analyses. Task speeds were calculated as $\log (1 / \mathrm{RT})$, where $\mathrm{RT}$ is response time in seconds. We computed the payoff sensitive performance response (Perf) as the difference in proportion selection of the RareG door between the $\Delta$ payoff and the $\Delta_{0}$ payoff blocks; RareG vs. RareL EVs differed only in the $\Delta$ payoff block. We computed gain frequency bias (Bias) as the difference in selected proportion of RareL and RareG doors in the $\Delta_{0}$ payoff block where the payoff for both the doors was the same. While Perf is indicative of subjective payoff maximization based selection of advantageous choices, Bias informs the choice bias and melioration for higher immediate gain frequencies. For $\mathrm{N}$ fraction of responses in each block, we calculated:

Perf $=N_{\text {exptRareG }}-N_{\text {baseRareG }}$

Bias $=N_{\text {baseRareL }}-N_{\text {baseRareG }}$

We also calculated Win-Stay and Lose-Shift performance on both $\Delta$ payoff and $\Delta_{0}$ payoff blocks. Win-Stay was computed as the proportion of times the subject repeated the same choice option in the next trial after obtaining a gain for choosing that option in the current trial. Lose-Shift was computed as the proportion of times the subject would shift away from the current choice option in the next trial, on obtaining a loss in the current trial.

Reinforcement Learning (RL) Model. We simulated three parsimonious RL models with up to two free parameters, which have a key role in reward-based decision-making, to explain the 185 2014; Sutton \& Barto, 1998):

186 1) Model A optimized the time scale of reward prediction $(\gamma)$ for every subject;

187 2) Model B optimized risk sensitivity $(\alpha)$ for every subject; and

188 3) Model $\mathrm{C}$ optimized both $\gamma$ and $\alpha$ to for every subject.

189 The time scale of reward prediction parameter $(\gamma)$ represents whether reward prediction is myopic 190 or long-sighted, lower values $\gamma \in\left(\begin{array}{ll}0 & 1\end{array}\right), \gamma \rightarrow 0$ suggest myopic reward prediction leading to 
impulsive decisions while higher values, $\gamma \rightarrow 1$ suggest long-sighted integration of rewards for decisions.

The risk sensitivity parameter $(\alpha)$ measures the extent to which expected uncertainty associated with the door influences the decision utility, the smaller the parameter value $\alpha \in(-11), \alpha \rightarrow-1$ the higher is risk seeking, while a larger value, $\alpha \rightarrow 1$ indicates high risk aversiveness.

The simulation agent had reward distributions as in the real experiment but scaled down by multiplying with a parameter 0.1 , and varying with blocks ( $\Delta$ payoff, $\Delta_{0}$ payoff) that were randomly ordered. There were as high as 50,000 trials in each block for letting model performance converge.

The agent has to choose between two doors each of which (stimulus, $s$ ) was represented by a radial basis function $(\Phi \mathrm{i})$ as below:

$$
\Phi s=\exp \frac{-\left(x-\mu_{s}\right)^{2}}{\sigma^{2}}
$$

Here, the $\mu_{\mathrm{s}}$ and $\sigma$ denotes the mean $\left(s \in\left[\begin{array}{ll}1 & 2\end{array}\right]\right.$; door $1=1$; door2 $=2$ ) and standard deviation of the inverse attention parameter, respectively. $\sigma$ is set to 1 in our models.

The door stimulus is multiplied with the weight matrix $w v$ for computing its value function, $Q$, and $w r$ for constructing its risk function, $\sqrt{ } h$.

Utility associated with any state at a trial, $t$, is the combination of value and risk function(Bell, 1995; d'Acremont et al., 2009), where the risk function is modulated by a risk sensitivity parameter $\alpha$. Higher the $\alpha$, higher the risk aversiveness of the subject.

$$
U(s, t)=Q(s, t)-\alpha \sqrt{h(s, t)}
$$

Where

$$
\begin{aligned}
& Q(s, t)=w v(s, t) \Phi(s) \\
& h(s, t)=w r(s, t) \Phi(s)
\end{aligned}
$$

The door choice selection is performed using the SoftMax principle defined as below. According to SoftMax, the probability for choosing a door at trial, $t$, is $P(\mathrm{~s}, \mathrm{t})$ :

$$
P(s, t)=\frac{\exp (\beta U(s, t))}{\sum_{i=1}^{n} \exp (\beta U(i, t))}
$$

Here, $n$ is the total number of doors available, and $\beta$ is the exploration index. $\beta$ is set to 1 in our models.

After choice selection, the weight functions are updated using the below principles. The choice value function $Q$ at trial $t+1$ for door, $s$, may be expressed as,

$$
Q_{t+1}(s, t)=Q_{t}(s, t)+\eta_{Q} \delta \Phi(s)
$$

where $\eta Q$ is the learning rate of the value function $(0<\eta Q<1)$ for the stimulus variable, $\Phi(\mathrm{s}) . \delta$ is the temporal difference error represented as

$$
\delta=r+\gamma \max _{s^{\prime}} Q\left(s^{\prime}, t\right)-Q_{t}(s, t)
$$

where $r$ is the reward associated with taking an action, a, for stimulus, $\mathrm{s}$, at time, $\mathrm{t}$, and $\gamma$ is the 
time scale of reward prediction. Similar to the value function, the risk function $h$ has an incremental update as defined by the below equation. Optimizing the risk function in addition to the value function is shown to capture human behavior well in a variety of cognitive tasks involving rewards, punishments and risk (Balasubramani, Chakravarthy, Ravindran, et al., 2015; Balasubramani et al., 2014).

$$
h_{t+1}(s, t)=h_{t}(s, t)+\eta_{h} \xi \Phi(s)
$$

where $\eta h$ is the learning rate of the risk function $(0<\eta h<1)$, and $\xi$ is the risk prediction error expressed by the below equation.

$$
\xi=\delta^{2}-h_{t}(s, t)
$$

For simplicity, we model as $\eta_{h}=\eta_{Q}=0.1$ as an initial optimization for our subjects for $\eta$ provided a median of 0.1 . The weights $w v$ and $w r$ are set to a small random number from set [-0.0005 0.0005] at trial $=1$. The weights are normalized by dividing by their norm.

The cost function optimizes the frequency of selections of rare gain and rare loss options in

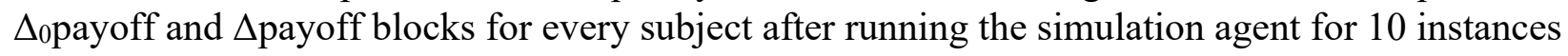
of 100,000 each, and inferring the optimal parameters for every participant in our study using fmincon function in MATLAB. Cost function $=$ sum of squares of the difference for observed actual ( Proportion\# RareG $\mathrm{expt}+$ Proportion\# RareL $\mathrm{L}_{\mathrm{exp}}+$ Proportion\# RareG $\mathrm{G}_{\mathrm{base}}+$ Proportion\# RareL $L_{\text {base}}$ ) - simulated actual (Proportion\# RareG $\mathrm{expt}+$ Proportion\# RareLexpt + Proportion\# Rare $_{\text {base }}+$ Proportion\# RareL $\mathrm{L}_{\text {base }}$ ). Optimization is carried out for either one (Models A,B) or two (Model C) parameters, using fmincon(). We ran fmincon() 100 times to choose the parameter set with least cost for any subject.

The AIC (Akaike Information Criteria) for these models were built using the likelihood function, which was estimated as the average correlation coefficient between the simulated and the observed key task behaviors - 1) payoff performance or Perf measure (eqn. 1); 2) gain frequency Bias measure (eqn. 1); and 3) the block differences between $\Delta$ payoff and $\Delta 0$ payoff blocks in Win-Stay results for the RareG door.

Neural data processing. We applied a uniform processing pipeline to all EEG data acquired simultaneous to the reward task. This included: 1) data pre-processing, 2) computing event related spectral perturbations (ERSP) for all channels, and 3) cortical source localization of the EEG data filtered within relevant theta, alpha and beta frequency bands.

1) Data preprocessing was conducted using the EEGLAB toolbox in MATLAB(Delorme \& Makeig, 2004). EEG data was resampled at $250 \mathrm{~Hz}$, and filtered in the 1-45 Hz range to exclude ultraslow DC drifts at $<1 \mathrm{~Hz}$ and high-frequency noise produced by muscle movements and external electrical sources at $>45 \mathrm{~Hz}$. EEG data were average referenced and epoched to the chosen door presentation during the task, in the $-.5 \mathrm{sec}$ to $+1.5 \mathrm{sec}$ time window (Figure 1). Any missing channel data (one channel each in 6 participants) was spherically interpolated to nearest neighbors. Epoched data were cleaned using the autorej function in EEGLAB to remove noisy trials $(>5 \mathrm{sd}$ outliers rejected over max 8 iterations; $0.91 \pm 2.65 \%$ of trials rejected per participant). EEG data 
were further cleaned by excluding signals estimated to be originating from non-brain sources, such as electrooculographic, electromyographic or unknown sources, using the Sparse Bayesian learning (SBL) algorithm(Ojeda et al., 2018, 2021), https://github.com/aojeda/PEB) explained below in the cortical source localization section.

2) For ERSP calculations, we performed time-frequency decomposition of the epoched data using the continuous wavelet transform (cwt) function in MATLAB's signal processing toolbox. Baseline time-frequency (TF) data in the $-250 \mathrm{~ms}$ to $-50 \mathrm{~ms}$ time window prior to chosen door presentation were subtracted from the epoched trials (at each frequency) to observe the eventrelated synchronization (ERS) and event-related desynchronization (ERD) modulations (Pfurtscheller, 1999)..

3) Cortical source localization was performed to map the underlying neural source activations for the ERSPs using the block-Sparse Bayesian learning (SBL) algorithm (Ojeda et al., 2018, 2021) implemented in a recursive fashion. This is a two-step algorithm in which the first-step is equivalent to low-resolution electromagnetic tomography (LORETA) (Pascual-Marqui et al., 1994). LORETA estimates sources subject to smoothness constraints, i.e. nearby sources tend to be co-activated, which may produce source estimates with a high number of false positives that are not biologically plausible. To guard against this, SBL applies sparsity constraints in the second step wherein blocks of irrelevant sources are pruned. This data-driven sparsity constraint of the SBL method reduces the effective number of sources considered at any given time as a solution, thereby reducing the ill-posed nature of the inverse mapping. In other words, one can either increase the number of channels used to solve the ill-posed inverse problem or impose more aggressive constraints on the solution to converge on the source model. The two-stage SBL produces evidence-optimized inverse source models at $0.95 \mathrm{AUC}$ relative to the ground truth while without the second stage $<0.9$ AUC is obtained (Ojeda et al., 2018, 2021). Prior research has also shown that sparse source imaging constraints can be soundly applied to low channel density data (Ding \& He, 2008; Stopczynski et al., 2014). Furthermore in Balasubramani et al. (42) we have shown that the ROI estimates resulting from this cortical source mapping have high test-retest reliability (Cronbach's alpha $=0.77, \mathrm{p}<0.0001$ ).

Source space activity signals were estimated and their root mean squares were partitioned into (1) regions of interest (ROIs) based on the standard 68 brain region Desikan-Killiany atlas (Desikan et al., 2006), using the Colin-27 head model (Holmes et al., 1998) and (2) artifact sources contributing to EEG noise from non-brain sources such as electrooculographic, electromyographic or unknown sources; activations from non-brain sources were removed to clean the EEG data. Cleaned subject-wise trial-averaged EEG data were then specifically filtered in theta $(3-7 \mathrm{~Hz})$, alpha $(8-12 \mathrm{~Hz})$, and beta $(13-30 \mathrm{~Hz})$ bands and separately source localized in each of these bands to estimate their cortical ROI source signals. The source signal envelopes were computed in MatLab (envelop function) by a spline interpolation over the local maxima separated by at least one time sample; we used this spectral amplitude signal for all neural analyses presented here. For analyses, we focused on theta, alpha and beta signals in the relevant selected choice period (0-500 ms after selected choice presentation), trial reward period (500$1000 \mathrm{~ms}$ after selected choice presentation), and the cumulative reward period (1000-1500 ms after selected choice presentation). 
315 Statistical Analyses. We fit robust multivariate linear regression models in MATLAB to 316 investigate the behavioral relationships between the Perf measure and demographic variables (age, 317 sex, race, ethnicity and SES), while controlling for Bias and order of block presentation. For any 318 linear regression model, the response variable was log-transformed for normality and we identified 319 significant factors contributing to the main effects. For regression models, we report the overall 320 model $\mathrm{R}^{2}$ and $\mathrm{p}$-value, and individual variable $\beta$ coefficients, $\mathrm{t}$-statistic, degrees of freedom, and 321 p-values.

Channel-wise theta, alpha, beta ERS and ERD modulations for significant spectral activity were computed relative to baseline by first processing for any outliers; any activations greater than $5 \mathrm{MAD}$ from the median were removed from further analyses. The significant average activity across all trials were found by performing t-tests $(\mathrm{p}<0.05)$ across subjects, followed by false discovery rate $(\mathrm{FDR}$, alpha $=0.05)$ corrections applied across the three dimensions of time, frequency, and channels (Genovese et al., 2002).

For computing source level activity correlates of the behavioral Perf measure, we first found the difference in RareG door specific neural activations between $\Delta$ payoff and $\Delta_{0}$ payoff blocks in three frequency bands - theta, alpha and beta and in three relevant trial periods: selected choice presentation, trial reward and cumulative reward period. We again used robust linear regression fits for identifying individual ROIs that relate to the Perf measure, while also accounting for individual Bias and variations in RL as predicted by the best fitting RL model. The results were family-wise error rate corrected for multiple comparisons for 3 trial periods and 3 frequency bands (FWER correction, $\mathrm{p}<0.0055$ ). The independently identified ROIs were further factored in a unified multivariate linear regression model to account for comparisons across ROIs; significant ROIs in this final multivariate model were reported after controlling for Bias, and the RL modelfit parameter $(\mathrm{p}<0.05)$.

Finally, we used robust multivariate linear regressions to model the self-reported mood symptoms of Anxiety, Depression, Inattention, Hyperactivity using predictors of demographic variables, Perf, Bias, RL model-fit parameter along with the identified neural correlates of Perf. Adjusted responses from robust multivariate models were plotted using the plotAdjustedResponse function in MATLAB. 


\section{Results}

Options associated with larger payoff show win-stay behavior and their choice fraction is proportional to individual gain frequency bias.

Healthy adult subjects $(\mathrm{N}=186$, ages $18-80$ years, 115 females $)$ performed a two-choice gambling task, Lucky Door, which implemented two distinct blocks of choices; the $\Delta_{\text {opayoff block }}$ delivered choice-sets with different gain frequencies but no differences in payoff, while the $\Delta$ payoff block delivered choice-sets with same gain frequencies as the $\Delta_{0}$ payoff block yet with

357 long-term payoff (i.e. expected value) differences (Figure 1A; Supplementary Table 1).

358 Specifically, the $\Delta_{0}$ payoff block only varied the gain frequency associated with the choice doors,

359 with one door leading to $70 \%$ positive reward outcomes (Rare Loss or RareL door) while the other

360 resulting in $70 \%$ negative reward outcomes (Rare Gain or RareG door), yet maintaining the same

361 reward average or long-term expected value/payoff. The $\Delta$ payoff block, presented in a random

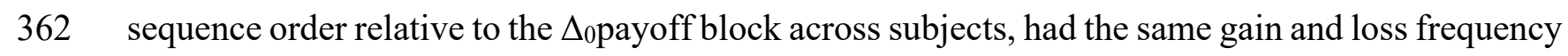

363 setup as the $\Delta_{0}$ payoff block, but the rewards associated with the RareG door resulted in a larger

364 long-term payoff than the RareL door. Participants executed 40 trials per block. Thus, we

365 calculated the payoff related performance measure, Perf as the difference in probability of RareG

366 selections on $\Delta$ payoff vs. $\Delta_{0}$ payoff blocks. Gain frequency Bias was measured as the difference in

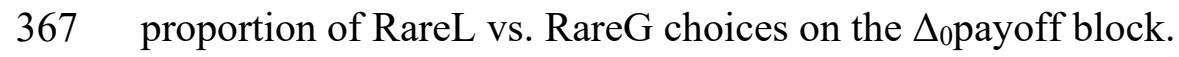

In terms of subject behavior, the actual proportion of RareG choices between the two

369 blocks did not differ (paired Wilcoxon signed-rank test: $\mathrm{N}_{\text {RareG }} \Delta$ payoff:17.74 \pm 0.39 ;

$370 \Delta_{0}$ payoff:18.47 $\left.\pm 0.40 ; \mathrm{p}=0.07\right)$. But, we found that Win-Stay behavior for the RareG choices

371 distinguished performance on the two blocks (mean \pm SEM Win-Stay RareG, $\Delta$ payoff:0.61 \pm 0.02 ; 


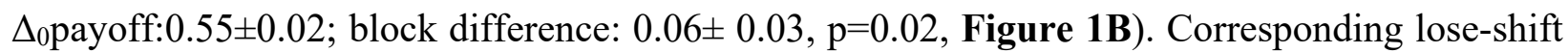
behavior did not differ between blocks $(\mathrm{p}=0.33)$.

Additionally, we found that the payoff-based responses, Perf were significantly correlated to individual gain frequency Bias $(\mathrm{r}=0.65, \mathrm{p}<0.0001$, Figure 1C).

Next, we implemented multivariate regression to model the payoff-related performance,

377
A

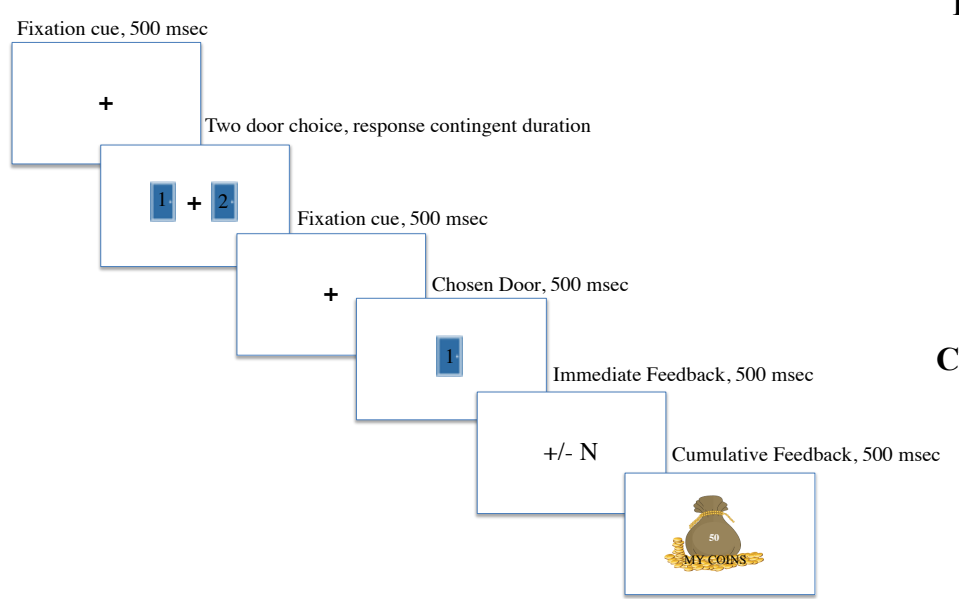

B $\quad 0.8 \quad$ RareG WinStay

C
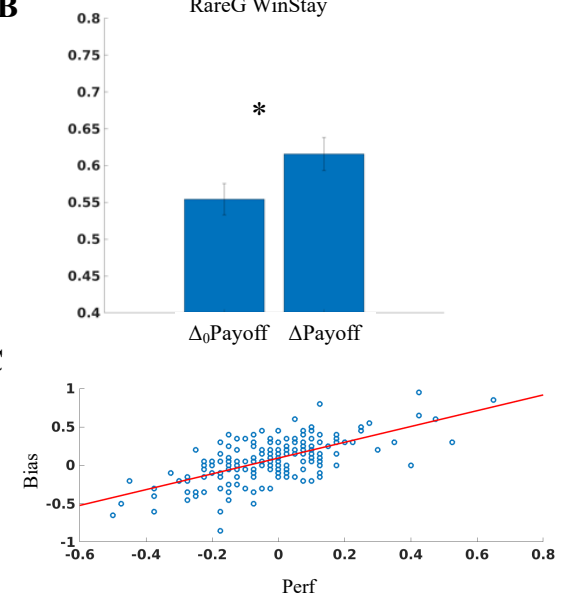

Figure 1. Reward task and associated behavior A) As per the task schematic, participants fixated for $0.5 \mathrm{sec}$, then chose from one of two choice doors. Post-response, fixation reappeared for $0.5 \mathrm{sec}$, followed by presentation of the chosen door for $0.5 \mathrm{sec}$, then immediate gain or loss feedback provided for $0.5 \mathrm{sec}$, and finally, cumulative feedback of all gains/losses up to the present trial shown for $0.5 \mathrm{sec}$. Reward distributions for the door choices are presented in Supplementary Table 1. B) Win-Stay behavior for the rare gain RareG door is significantly greater on the $\Delta$ Payoff versus $\Delta_{0}$ Payoff block. ${ }^{*}: \mathrm{p}<.05$. C) Gain frequency Bias significantly predicts payoff performance, $\operatorname{Perf}(\mathrm{r}=0.65, \mathrm{p}<0.0001)$. 


\begin{tabular}{|c|c|}
\hline Demographics & Median \pm MAD \\
\hline Age & $25.00 \pm 14.87$ \\
\hline \multicolumn{2}{|l|}{ Gender n (\%) } \\
\hline Male & $71(38.17)$ \\
\hline Female & $115(61.83)$ \\
\hline \multicolumn{2}{|l|}{ Ethnicity n (\%) } \\
\hline Caucasian & $116(62.37)$ \\
\hline Black or African American & $4(2.15)$ \\
\hline Native Hawaiian, Pacific Islander & $0(0)$ \\
\hline Asian & $37(19.89)$ \\
\hline American Indian, Alaska Native & $4(2.15)$ \\
\hline Multi-racial & $12(6.45)$ \\
\hline Others & $12(6.45)$ \\
\hline \multicolumn{2}{|l|}{ Race n (\%) } \\
\hline Hispanic or Latino & $25(13.51)$ \\
\hline Not Hispanic or Latino & $155(83.78)$ \\
\hline Unknown & $5(2.70)$ \\
\hline SES & $5.00 \pm 1.34$ \\
\hline Mental Health & Median \pm MAD \\
\hline Anxiety & $3 \pm 2.88$ \\
\hline Depression & $3 \pm 2.76$ \\
\hline Inattention & $4 \pm 3.99$ \\
\hline Hyperactivity & $3 \pm 2.96$ \\
\hline Behavior & Median \pm MAD \\
\hline Perf & $-0.02 \pm 0.13$ \\
\hline Bias & $0.10 \pm 0.21$ \\
\hline Model $\gamma$ & $0.99 \pm 0.07$ \\
\hline
\end{tabular}

Table 1. Subject characteristics. Median \pm MAD for subjects demographics variables, mental health self-report scores, and parameter $\gamma$ from the preferred reinforcement learning model. MAD: median absolute deviation, SES: socioeconomic status score.

401 Individual differences in reward prediction explain task behavior.

Prior RL modeling studies have suggested that task behavior can be explained with greater accuracy when both the long term expected value or payoff and the expected risk are accounted

404 for during decision-making (Balasubramani, Chakravarthy, Ravindran, et al., 2015;

405 Balasubramani et al., 2014). The major advantages of building these RL models are that the models

406 can characterize the individual differences in each participant in terms of computational 
407 parameters that manifest in learning and executive control. Secondly, they can explain the

408

409

410

411

412

413 414 reinforcement learning models (RL, Figure 2A) that simulate behavior by optimizing time scale converged behavioral dynamics in each participant sans experimental trial limitations.

Using this RL framework, we were interested in investigating how are choice decisions affected by 1) extent of integration of rewards over time i.e. time scale of reward prediction, and 2) differential risk sensitivity to gains and losses affecting the choices, where risk is the variance in reward outcomes. In order to find whether the observed subjective behavioral differences are driven by one of the two or both of the above decision making measures, we built three separate of reward prediction ( $\gamma$ : model A), risk sensitivity ( $\alpha$ : model B) or both $\gamma$ and $\alpha$ parameters (model C). In model A, higher $\gamma$ parameter values represent long-sightedness; risk sensitivity $\alpha$ is set to 0 in this model. In model B, higher $\alpha$ parameter values indicate risk aversiveness and lower values indicate risk seeking; the reward prediction $\gamma$ parameter is set to 0 in this model. All models were optimized using robust solvers in MATLAB (see Methods to find global minima with multiple random initial conditions) to match to the observed choice selection behavior observed in each block.

Next, we computed the likelihood of any Model A, B, C to explain the significantly observed behavioral measures: which are Perf, Bias and the block differences in proportion of Win-Stay to the RareG door. The likelihood, taken as correlations between the simulated and actually observed, was used to construct model AIC (Akaike Information Criterion). The AIC values for Models A, B and C were 2.94, 3.70 and 5.45, respectively (Figure 2B), suggesting that Model A can preferably explain the observed behavior (Figure 2C). This means the individual differences in the time scale of reward prediction can preferably explain the reward maximization (Perf) as well as melioration (Bias) behavior. On parameter recovery analysis for this model, the 
430 simulated model solutions were significantly correlated with the recovered solutions, implying that

431 our model can reliably explain the subjective behaviors (Wilson \& Collins, 2019).

A

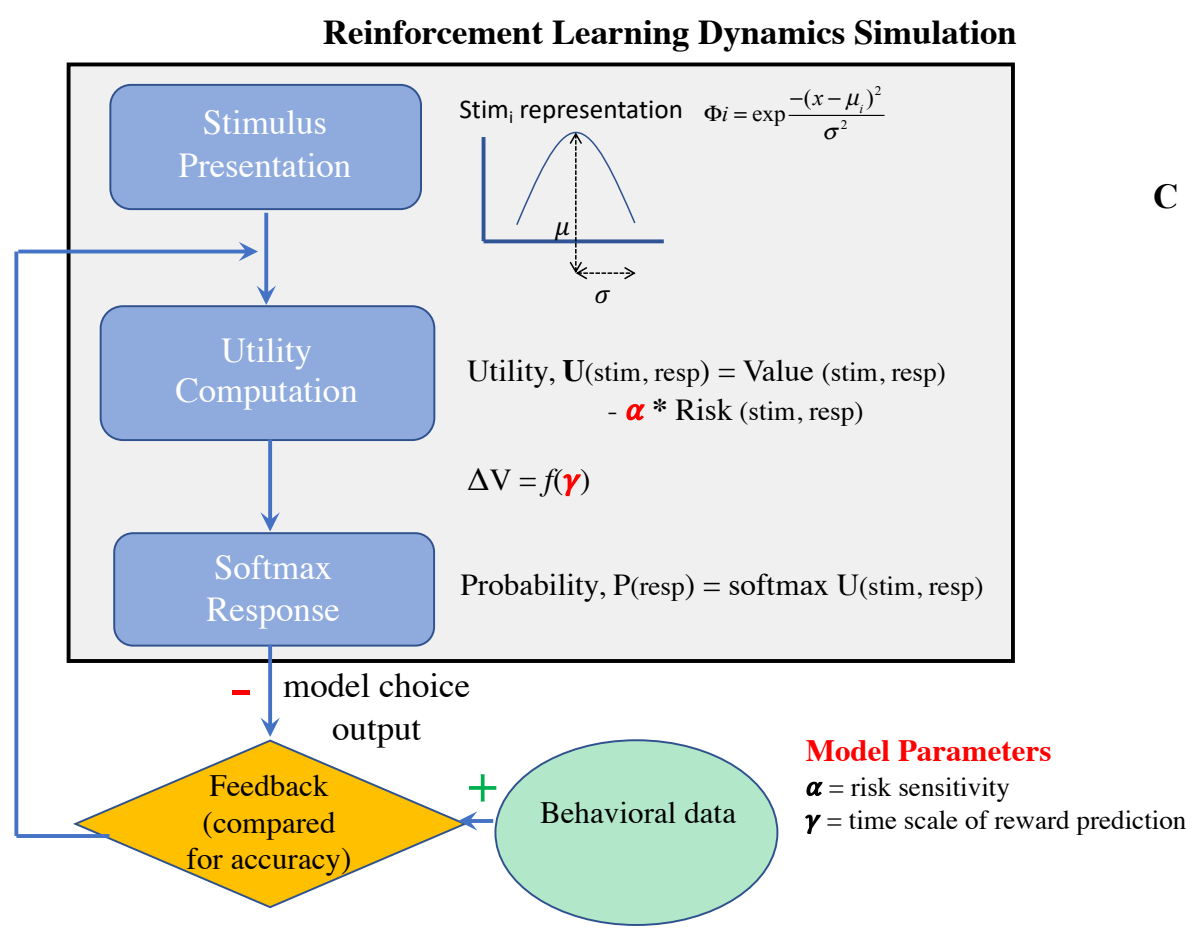

432

433

434

435

436

437

438

439

440

441

442

443

444

445

446
B

C
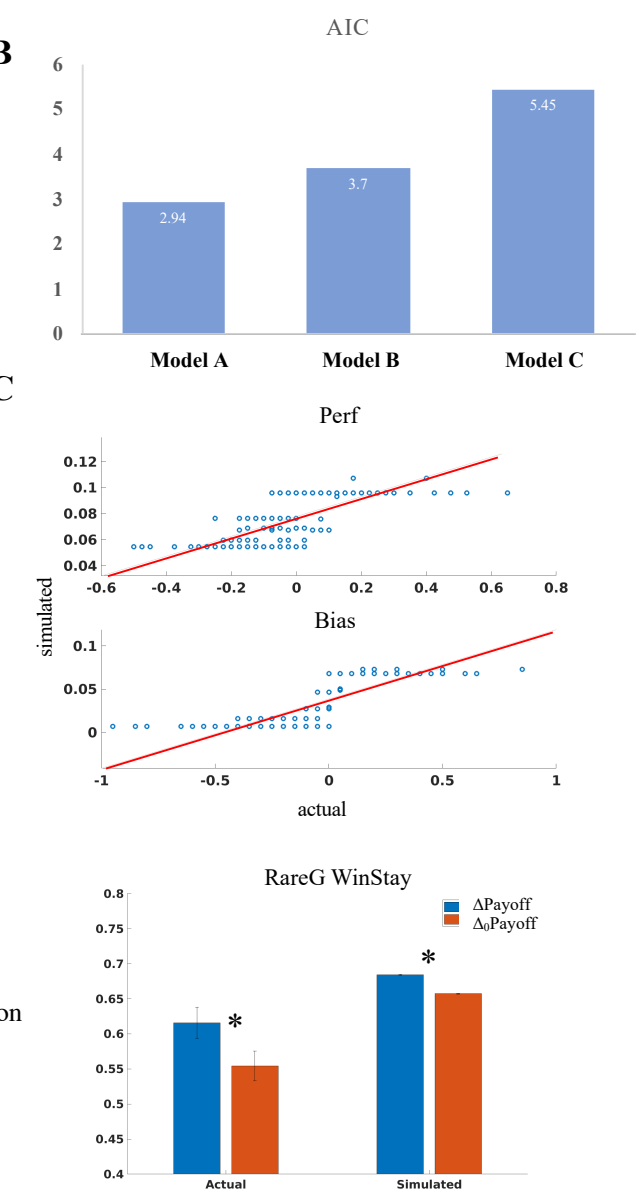

Figure 2. Reinforcement Learning Model (A) Schematic representing the stimulus, value function and choice selection modules. The model results for number of selections associated with each of the choice door stimuli in each task block are compared against the actual selections made by each subject, for purposes of model optimization. The model uses the utility, U, associated with each choice response for making the decision, where the utility is a function of reward average and reward variance associated with choices. The decision in the model is taken using the SoftMax probability, P, of making the choices. Model parameters are highlighted as $\alpha$ (model agent's differential risk sensitivity to gain and loss outcome uncertainties), and $\gamma$ (time scale of reward prediction). (B) AIC values for three models, Model A ( $\gamma$ optimized), B ( $\alpha$ optimized), C ( $\gamma$ and $\alpha$ optimized), show that the $\gamma$ model was best performing. (C) The $\gamma$ optimized model showed strong correlations between simulated and actual Perf $(\rho(185)=0.81, p<0.0001)$, Bias $(\rho(185)=0.89$, $\mathrm{p}<0.0001)$ and RareG Win-Stay difference between blocks $(\rho(185)=0.17 \mathrm{p}=0.004)$. 


\section{Right rostral anterior cingulate cortex encodes reward payoff maximization.}

Participants performed the reward task with simultaneous EEG, which we analyzed in the

theta $(3-7 \mathrm{~Hz})$, alpha $(8-12 \mathrm{~Hz})$, and beta $(13-30 \mathrm{~Hz})$ frequency bands in cortical source space

450 parcellated as per the Desikan-Killiany regions of interest (Desikan et al., 2006). To identify the

451 neural correlates underlying reward payoff maximization (Perf), we modeled the neural variables

452 as predictors of Perf using robust multivariate linear regression, while accounting for gain

453 frequency Bias that was significantly related to Perf (Figure 1), and the optimal RL model

$454 \operatorname{parameter}(\gamma)$

We investigated neural activations from three relevant trial periods: immediately post-

456 presentation of selected choice but prior to reward (0-500 ms selected choice period), during

457 presentation of trial reward (0-500 ms reward period), and during presentation of the cumulative

458 reward up to that trial in the trial sequence (0-500 ms cumulative reward period). Perf neural

459 activations were the relative difference in activity on $\Delta$ payoff vs. $\Delta_{0}$ payoff block RareG trials.

460 Taking the relative block difference allowed non-task related individual EEG differences to cancel

461 out. Relative responses to the RareG door were important for analysis because this door choice

462 resulted in a larger long-term payoff than the other (RareL) door in the $\Delta$ payoff block. We applied

463 family-wise error-rate (fwer) corrections to the Perf source-space neural correlates to account for

464 multiple comparisons across three frequency bands (theta, alpha, beta) and three time periods

465 (choice, reward, cumulative reward). Figure 3A shows the Perf source-space neural correlates

466 found to be significant in this analysis; all neural activations inversely related to Perf.

We further accounted for the multiple independently significant cortical ROI predictors

468 (Figure 3A) within a unified multivariate model for Perf that also included the significant Bias and 469 RL model parameter $\gamma$ as covariates. This overall multivariate model for Perf was significant 
$470 \quad\left(\mathrm{R}^{2}=0.69, \mathrm{df}=126, \mathrm{p}<0.0001\right)$. The results of this model showed alpha activity in the bilateral

471 rostral anterior cingulate cortex (rACC) during the reward period (left: $\beta=-394.32 \pm 125.56$,

$472 \mathrm{t}(177)=-3.14, \mathrm{p}=0.002$; right: $\beta=-634.89 \pm 220.51, \mathrm{t}(176)=-2.88, \mathrm{p}=0.004)$ and theta activity in the

473 right $\mathrm{rACC}$ during the cumulative reward period $(\beta=-56.98 \pm 17.93, \mathrm{t}(177)=-3.17, \mathrm{p}=0.002)$ as the

474 most significant predictors of payoff-based decisions; activity in the selected choice presentation

475 period did not survive the multivariate model. We then investigated whether these specific

476 theta/alpha rACC activations also related to gain frequency Bias, while controlling for the Perf

477 payoff. The bilateral alpha band rACC activations were significantly related to Bias (left: $\beta=-$

$478514.67 \pm 205.05, \mathrm{t}(178)=-2.51, \mathrm{p}=0.01$; right: $\beta=-886.15 \pm 348.74, \mathrm{t}(179)=-2.54, \mathrm{p}=0.01)$ but not

479 right $\mathrm{rACC}$ theta $(\mathrm{p}>0.05)$. Thus, right $\mathrm{rACC}$ theta activity during the cumulative reward period

480 was the distinct neural correlate of reward payoff (Figure 3B); corresponding scalp theta activity

481 and topography are shown in Figure 3C.

482 Additionally, we checked whether the distinct Perf correlate of right rACC theta activation

483 during the cumulative reward period showed any interactions with age and gender, but no

484 significant interactions were found $(\mathrm{p}>0.1)$.

Distinct neural correlates of reward maximization in right $\mathrm{rACC}$ predict depressed mood.

Finally, we investigated whether the neural correlates of payoff decisions are relevant to

488 subjective mental health by modeling anxiety, depression, inattention and hyperactivity self-

489 reported mood symptoms scores as dependent variables in robust multivariate regression models.

490 All demographic variables (age, gender, race, ethnicity, SES), task performance variables of Perf

491 and Bias, the relevant RL model parameter $\gamma$, as well as the reward-processing neural correlates 
503
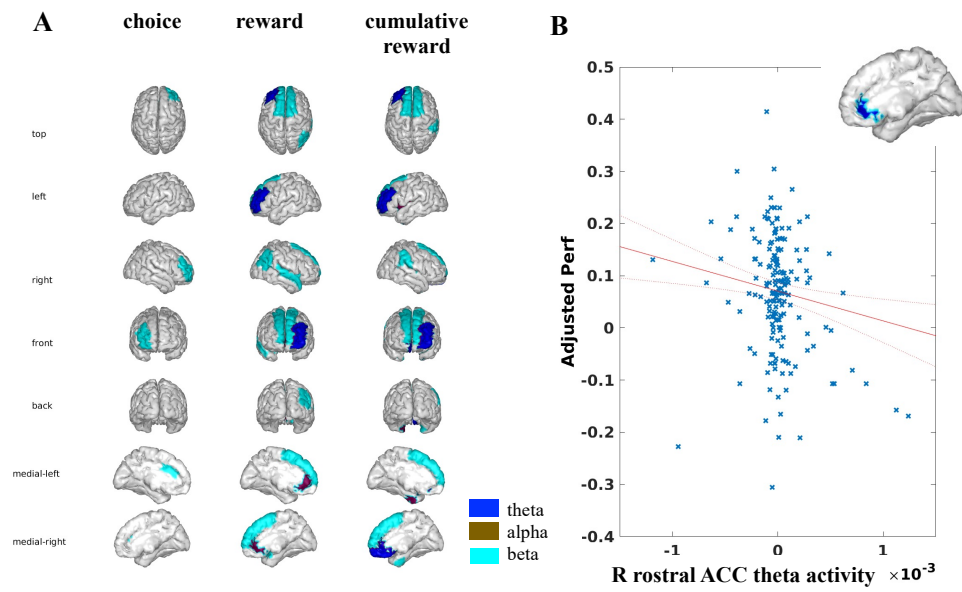

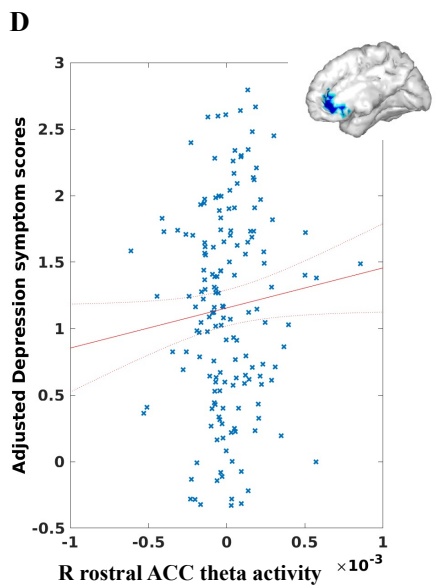

Figure 3: Neural correlates of payoff-based decision making in humans. (A) Independent neural correlates in theta (blue), alpha (brown), and beta (cyan) bands found to be inversely related to Perf in the choice selection, reward and cumulative reward epochs $(p<0.0055)$. (B) Only right rostral anterior cingulate cortex (rACC) theta activity in the cumulative reward period uniquely relates to Perf after controlling for Bias and time scale of reward prediction. (C) Corresponding theta band temporal activity on RareG trials at mid-frontal scalp channel AFz showing differences between payoff blocks (top), and scalp topography of RareG theta band trial

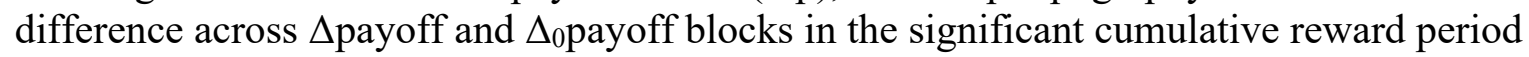
(bottom). (D) Perf related rACC theta positively predicts depressed mood symptoms. The scatter plots in (B) and (D) are presented on an adjusted axis as obtained from the multivariate robust regression models; the $\mathrm{x}$-axes for these plots are $10^{-3}$ source activity units. 
Discussion

Reinforcement learning models suggest healthy human choices ideally tend to maximize

521 Gupta et al., 2013). However, many existing neuropsychological measures of decision-making that

522 optimize for long-term payoffs don't reliably estimate the participant's ability to integrate rewards

523 and make foresighted decisions, and instead suffer from biased predispositions (Furl, 2010;

524 Gansler et al., 2011; Napoli \& Fum, 2010; Stocco et al., 2009); this tendency to choose lesser local

525 minima based gain over long-term gain is also referred to as melioration (Sims et al., 2013). In our

526 paradigm, we study gain frequency driven bias separate from payoff-based decision-making by

527 introducing a $\Delta_{0}$ payoff block with no payoff difference between choice options wherein decisions

528 are purely based on gain frequency (Lin et al., 2009). Comparing choices within the $\Delta$ payoff

529 experimental block, designed to have similar reward distribution structure as the $\Delta_{0}$ payoff block

530 but differing only on the long-term outcome between options, allows measurement of long-term

531 payoff maximization strategy. Therefore, our study design is able to distinguish reward

532 maximization from melioration and further leverages these measures to inform mental health

533 behaviors.

The behavioral outcomes of our experiment varied based on individual subject

535 characteristics. We found that payoff-based performance was significantly related to individual

536 bias for observed frequency of gains; this is in line with prior studies of decision-making but

537 wherein gain frequency decisions are often conflated with expected value (Bechara et al., 1997;

538 Lin et al., 2009). We further modeled subjective differences in reinforcement learning based

539 decision making and by using RL modeling framework, we extracted subjective sensitivity to risks 
$540(\alpha)$ and time scale of reward prediction $(\gamma)$ to explain each subject's behavior. The parsimonious

541 RL models suggested that the observed behavior is preferably explained by the differences in the

542 extent of reward prediction over time $(\gamma)$ between individuals.

543 Uniquely, we then investigated the neural correlates of payoff performance while

544 accounting for individual differences in gain frequency bias and extent of reward prediction.

545 Earlier studies have suggested complex dynamics mediated by dopamine and serotonin

546 neuromodulation in the cortico-basal ganglia circuit to underlie reward and risk based decision

547 making (Balasubramani, Chakravarthy, Ali, et al., 2015b; Balasubramani, Chakravarthy, et al.,

548 2018; Balasubramani et al., 2014; Chakravarthy et al., 2018; Doya, 2002; Schultz, 2000; Sutton \&

549 Barto, 1998; Tobler et al., 2009). In our study, we focused on three different time periods of the

550 task, the first associated with processing of the selected choice, the ensuing reward presentation

551 period and the cumulative reward period to understand how neural dynamics in these periods affect

552 payoff-based performance. The selected choice period captures the processing associated with

553 presentation of the chosen door after the actual decision period. We did not analyze the actual

554 decision period since two different choice options are shown on the screen during this time and

555 the signal associated with every choice option was difficult to explicitly assess. These analyses

556 showed distinct neural correlates of payoff based performance in the core frontal executive region

557 of the right rACC during the cumulative reward period. Relatedly, most prior studies on

558 probabilistic reward processing have suggested this medial prefrontal cortex (mPFC) region to be

559 critical for mediating decision performance (Balasubramani \& Hayden, 2018; Bechara et al., 1997,

560 2000; Brevers et al., 2013; Kennerley et al., 2011; Moccia et al., 2017).

$561 \quad$ More specifically, analyses showed theta activity in right rACC negatively associated with

562 payoff-based performance. This finding is aligned with prior evidence for reward-based theta 
563 processing and its widely studied relationship to long-term risk or uncertainty (Cavanagh et al.,

564 2010; Christie \& Tata, 2009; Zavala et al., 2018). This may be one reason why we observe a 565 negative relationship between cumulative reward period rACC theta and effective payoff, Perf,

566 because long-term payoff magnitude has been shown to inversely relate to uncertainty but

567 positively to choice utility (Behrens et al., 2007; Krain et al., 2006; Paulus \& Frank, 2006;

568 Preuschoff et al., 2006). Notably, reward period bilateral rACC alpha also related to payoff

569 maximization, but this was not a distinct correlate of payoff as it also significantly explained gain

570 frequency bias or melioration in our task.

571 Translational neuroscience studies show that reward based decision processing deficits are

572 found in depression and in attention disorders, leading to difficulty in reward integration and

573 foresighted choice-behaviors (Balasubramani, Chakravarthy, Ali, et al., 2015b; Balasubramani \&

574 Chakravarthy, 2019; Gradin et al., 2011; Groen et al., 2013; Miller \& Seligman, 1973; Silvetti et

575 al., 2013; Ziegler et al., 2016). Such individuals then focus on the immediate reward outcome in

576 the short-term, characterized by a prolonged attenuation of temporal discounting of rewards (Eshel

577 \& Roiser, 2010; Pizzagalli et al., 2005). Interestingly, rACC theta activity in our paradigm during

578 the cumulative reward period, which negatively correlated with payoff performance, was a positive

579 predictor of depressed mood. This result is aligned with prior research using fMRI showing that

580 the volume and activation patterns of rACC correlates with depressed mood (Boes et al., 2008;

581 Yoshimura et al., 2010). Related research also suggests that rACC theta activity is a significant

582 baseline marker for depression treatment outcomes (Pizzagalli et al., 2018). These results are still

583 limited by our study in healthy adults and need to be replicated in clinical populations, and with

584 greater channel density electrophysiology recordings. 
Altogether, our study presents the importance of controlling for melioration biases for

586 immediate reward frequency and individual differences in learning while assessing advantageous,

587 i.e. foresighted, decision-making ability in humans. Our findings of payoff-relevant right rostral

588 ACC theta activity in the cumulative reward feedback period, could be important for clinical

589 translational application, particularly for depression, suggesting a plausible neural target for

590 interventions that engage reward processing.

591 
593 Supplementary Data

594

595

\begin{tabular}{ccccc}
\hline & \multicolumn{2}{c}{$\Delta$ payoff } & \multicolumn{2}{c}{$\Delta$ opayoff } \\
& RareL & RareG & RareL & RareG \\
\hline $\mathbf{1}$ & 20 & -20 & 30 & -30 \\
$\mathbf{2}$ & 20 & -20 & 30 & -30 \\
$\mathbf{3}$ & 20 & -20 & 30 & -30 \\
$\mathbf{4}$ & 20 & -20 & 30 & -30 \\
$\mathbf{5}$ & 20 & -20 & 30 & -30 \\
$\mathbf{6}$ & 20 & -20 & 30 & -30 \\
$\mathbf{7}$ & 20 & -20 & 30 & -30 \\
$\mathbf{8}$ & -60 & 60 & -70 & 70 \\
$\mathbf{9}$ & -60 & 60 & -70 & 70 \\
$\mathbf{1 0}$ & -60 & 60 & -70 & 70 \\
\hline sum & -40 & 40 & 0 & 0 \\
\hline average & -4 & 4 & 0 & 0 \\
\hline variance & 1493.33 & 1493.33 & 2333.33 & 2333.33 \\
\hline
\end{tabular}

597 Supplementary Table 1. Reward distributions for the door choices in the $\Delta$ payoff and $598 \Delta_{\mathbf{0}}$ payoff blocks. The two door choices in either block were RareG (rare gains and frequent losses) 599 and RareL (rare losses and frequent gains). Payoff (expected value) for RareG and RareL were the 600 same in the $\Delta_{0}$ payoff block and greater for RareG relative to RareL in the $\Delta$ payoff block. RareG 601 and RareL distributions had the same sum, average and variance in the $\Delta_{0}$ payoff block, and 602 different sum and averages but same variance in the $\Delta$ payoff block.

603 


\section{Acknowledgements}

605 This work was supported by University of California San Diego (UCSD) lab start-up funds (DR, $606 \mathrm{JM}$ ), the Interdisciplinary Research Fellowship in NeuroAIDS (PB: R25MH081482), the Brain \& 607 Behavior Research Fund (PB), the Kavli Foundation (PB, JM), and the Sanford Institute for 608 Empathy and Compassion (JM, PB). We thank Alankar Misra for software development of the 609 BrainE software and several UCSD undergraduate students who assisted with data collection. The 610 BrainE software is copyrighted for commercial use (Regents of the University of California 611 Copyright \#SD2018-816) and free for research and educational purposes. We thank Sabyasachi 612 Shivkumar and Vignesh Muralidharan for their helpful feedback on the study analysis.

613

\section{Data Availability}

615 A part of the dataset with 96 of the 186 participants data used in this study is available on the open616 access repository link: 10.5281/zenodo.4088951 


\section{References}

Admon, R., \& Pizzagalli, D. A. (2015). Dysfunctional reward processing in depression. Current

\section{3}

624

625

626

$$
\text { Opinion in Psychology, 4, 114-118. https://doi.org/10.1016/j.copsyc.2014.12.011 }
$$

Arevalo-Rodriguez, I., Smailagic, N., i Figuls, M. R., Ciapponi, A., Sanchez-Perez, E., Giannakou, A., Pedraza, O. L., Cosp, X. B., \& Cullum, S. (2015). Mini-Mental State Examination (MMSE) for the detection of Alzheimer's disease and other dementias in people with mild cognitive impairment (MCI). Cochrane Database of Systematic Reviews, 3.

Aron, A. R., Durston, S., Eagle, D. M., Logan, G. D., Stinear, C. M., \& Stuphorn, V. (2007). Converging Evidence for a Fronto-Basal-Ganglia Network for Inhibitory Control of Action and Cognition. Journal of Neuroscience, 27(44), 11860-11864. https://doi.org/10.1523/JNEUROSCI.3644-07.2007

Balasubramani, P. P., Chakravarthy, S., Ravindran, B., \& Moustafa, A. A. (2014). An extended reinforcement learning model of basal ganglia to understand the contributions of serotonin and dopamine in risk-based decision making, reward prediction, and punishment learning. Frontiers in Computational Neuroscience, 8, 47.

Balasubramani, P. P., Chakravarthy, S., Ravindran, B., \& Moustafa, A. A. (2015). A network model of basal ganglia for understanding the roles of dopamine and serotonin in rewardpunishment-risk based decision making. Name: Frontiers in Computational Neuroscience, 9, 76 .

Balasubramani, P. P., \& Chakravarthy, V. S. (2019). Bipolar oscillations between positive and negative mood states in a computational model of Basal Ganglia. Cognitive Neurodynamics. https://doi.org/10.1007/s11571-019-09564-7 
643 Balasubramani, P. P., Chakravarthy, V. S., Ali, M., Ravindran, B., \& Moustafa, A. A. (2015a). Impulsivity in Parkinson's Disease Patients. PLoS One, 10(6), e0127542.

Balasubramani, P. P., Chakravarthy, V. S., Ali, M., Ravindran, B., \& Moustafa, A. A. (2015b).

Balasubramani, P. P., Chakravarthy, V. S., Ravindran, B., \& Moustafa, A. A. (2018). Modeling Models of the Basal Ganglia (pp. 215-243). Springer.

Balasubramani, P. P., \& Hayden, B. (2018). Overlapping neural processes for stopping and economic choice in orbitofrontal cortex. BioRxiv, 304709.

Balasubramani, P. P., Moreno-Bote, R., \& Hayden, B. Y. (2018). Using a Simple Neural Network to Delineate Some Principles of Distributed Economic Choice. Frontiers in Computational Neuroscience, 12, 22.

Balasubramani, P. P., Ojeda, A., Grennan, G., Maric, V., Le, H., Alim, F., Zafar-Khan, M., DiazDelgado, J., Silveira, S., \& Ramanathan, D. (2020). Mapping Cognitive Brain Functions knowing the advantageous strategy. Science, 275(5304), 1293-1295.

663 Bechara, A., Tranel, D., \& Damasio, H. (2000). Characterization of the decision-making deficit of patients with ventromedial prefrontal cortex lesions. Brain, 123(11), 2189-2202. 
Behrens, T. E. J., Woolrich, M. W., Walton, M. E., \& Rushworth, M. F. S. (2007). Learning the value of information in an uncertain world. Nature Neuroscience, 10(9), 1214-1221. https://doi.org/10.1038/nn1954

Bell, D. E. (1995). Risk, return, and utility. Management Science, 41(1), 23-30.

Bembich, S., Clarici, A., Vecchiet, C., Baldassi, G., Cont, G., \& Demarini, S. (2014). Differences in time course activation of dorsolateral prefrontal cortex associated with low or high risk choices in a gambling task. Frontiers in Human Neuroscience, 8, 464.

Boes, A. D., McCormick, L. M., Coryell, W. H., \& Nopoulos, P. (2008). Rostral Anterior Biological Psychiatry, 63(4), 391-397. https://doi.org/10.1016/j.biopsych.2007.07.018

Brevers, D., Bechara, A., Cleeremans, A., \& Noel, X. (2013). Iowa Gambling Task (IGT): Twenty years after - gambling disorder and IGT. Frontiers in Psychology, 4. https://doi.org/10.3389/fpsyg.2013.00665

Cavanagh, J. F., Frank, M. J., Klein, T. J., \& Allen, J. J. (2010). Frontal theta links prediction errors to behavioral adaptation in reinforcement learning. Neuroimage, 49(4), 3198-

Chakravarthy, V. S., Balasubramani, P. P., Mandali, A., Jahanshahi, M., \& Moustafa, A. A. (2018). The many facets of dopamine: Toward an integrative theory of the role of dopamine in managing the body's energy resources. Physiology \& Behavior.

685 Chiu, Y.-C., \& Lin, C.-H. (2007). Is deck C an advantageous deck in the Iowa Gambling Task? 
Chiu, Y.-C., Lin, C.-H., Huang, J.-T., Lin, S., Lee, P.-L., \& Hsieh, J.-C. (2008). Immediate gain is long-term loss: Are there foresighted decision makers in the Iowa Gambling Task? Behavioral and Brain Functions, 4(1), 13.

Christakou, A., Brammer, M., Giampietro, V., \& Rubia, K. (2009). Right ventromedial and dorsolateral prefrontal cortices mediate adaptive decisions under ambiguity by integrating

Christie, G. J., \& Tata, M. S. (2009). Right frontal cortex generates reward-related theta-band oscillatory activity. Neuroimage, 48(2), 415-422.

d'Acremont, M., Lu, Z.-L., Li, X., Van der Linden, M., \& Bechara, A. (2009). Neural correlates of risk prediction error during reinforcement learning in humans. Neuroimage, 47(4),

Delorme, A., \& Makeig, S. (2004). EEGLAB: An open source toolbox for analysis of single-trial EEG dynamics including independent component analysis. Journal of Neuroscience Methods, 134(1), 9-21. https://doi.org/10.1016/j.jneumeth.2003.10.009 automated labeling system for subdividing the human cerebral cortex on MRI scans into gyral based regions of interest. NeuroImage, 31(3), 968-980. 
710 Dillon, D. G., Rosso, I. M., Pechtel, P., Killgore, W. D., Rauch, S. L., \& Pizzagalli, D. A. (2014).

711 Peril and pleasure: An RDOC-inspired examination of threat responses and reward processing in anxiety and depression. Depression and Anxiety, 31(3), 233-249.

713 Ding, L., \& He, B. (2008). Sparse source imaging in electroencephalography with accurate field modeling. Human Brain Mapping, 29(9), 1053-1067.

715 Doya, K. (2002). Metalearning and neuromodulation. Neural Networks, 15(4-6), 495-506.

716 Eshel, N., \& Roiser, J. P. (2010). Reward and punishment processing in depression. Biological Psychiatry, 68(2), 118-124.

718 Franken, I. H., \& Muris, P. (2005). Individual differences in decision-making. Personality and Individual Differences, 39(5), 991-998.

Furl, B. A. (2010). The influence of individual differences on the Iowa Gambling Task and realworld decision making [PhD Thesis]. Wake Forest University.

722 Gansler, D. A., Jerram, M. W., Vannorsdall, T. D., \& Schretlen, D. J. (2011). Comparing alternative metrics to assess performance on the Iowa Gambling Task. Journal of Clinical and Experimental Neuropsychology, 33(9), 1040-1048. https://doi.org/10.1080/13803395.2011.596820

Garon, N., Moore, C., \& Waschbusch, D. A. (2006). Decision Making in Children With ADHD Only, ADHD-Anxious/Depressed, and Control Children Using a Child Version of the

730 Genovese, C. R., Lazar, N. A., \& Nichols, T. (2002). Thresholding of Statistical Maps in Functional Neuroimaging Using the False Discovery Rate. NeuroImage, 15(4), 870-878. https://doi.org/10.1006/nimg.2001.1037 
733 Gradin, V. B., Kumar, P., Waiter, G., Ahearn, T., Stickle, C., Milders, M., Reid, I., Hall, J., \& schizophrenia. Brain, 134(6), 1751-1764. https://doi.org/10.1093/brain/awr059

Groen, Y., Gaastra, G. F., Lewis-Evans, B., \& Tucha, O. (2013). Risky behavior in gambling tasks in individuals with ADHD-a systematic literature review. PLoS One, 8(9), e74909.

Gupta, A., Balasubramani, P. P., \& Chakravarthy, S. (2013). Computational model of precision grip in Parkinson's disease: A Utility based approach. Frontiers in Computational Neuroscience, 7. https://doi.org/10.3389/fncom.2013.00172

Gupta, R., Koscik, T. R., Bechara, A., \& Tranel, D. (2011). The amygdala and decision-making.

Hampshire, A., Chamberlain, S. R., Monti, M. M., Duncan, J., \& Owen, A. M. (2010). The role of the right inferior frontal gyrus: Inhibition and attentional control. NeuroImage, 50(3).

Harman, J. L. (2011). Individual differences in need for cognition and decision making in the

Holmes, C. J., Hoge, R., Collins, L., Woods, R., Toga, A. W., \& Evans, A. C. (1998). Computer Assisted Tomography, 22(2), 324-333.

751 Kennerley, S. W., Behrens, T. E., \& Wallis, J. D. (2011). Double dissociation of value computations in orbitofrontal and anterior cingulate neurons. Nature Neuroscience,

754 Kothe, C., Medine, D., Boulay, C., Grivich, M., \& Stenner, T. (2019). “Lab Streaming Layer” 
Krain, A. L., Wilson, A. M., Arbuckle, R., Castellanos, F. X., \& Milham, M. P. (2006). Distinct neural mechanisms of risk and ambiguity: A meta-analysis of decision-making. NeuroImage, 32(1), 477-484. https://doi.org/10.1016/j.neuroimage.2006.02.047

Kroenke, K., Spitzer, R. L., \& Williams, J. B. W. (2001). The PHQ-9: Validity of a Brief Depression Severity Measure. Journal of General Internal Medicine, 16, 606-613.

Lin, C., Chiu, Y., \& Huang, J. (2009). Gain-loss frequency and final outcome in the Soochow Gambling Task: A Reassessment. Behavioral and Brain Functions, 5, 1-9. https://doi.org/10.1186/1744-9081-5-45

Luman, M., Tripp, G., \& Scheres, A. (2010). Identifying the neurobiology of altered reinforcement sensitivity in ADHD: A review and research agenda. Neuroscience \&

Miller, W. R., \& Seligman, M. E. (1973). Depression and the perception of reinforcement. Journal of Abnormal Psychology, 82(1), 62-73. https://doi.org/10.1037/h0034954

Moccia, L., Pettorruso, M., Crescenzo, F. D., Risio, L. D., Martinotti, G., Bifone, A., Janiri, L.,

Mueller, E. M., Nguyen, J., Ray, W. J., \& Borkovec, T. D. (2010). Future-oriented decisionmaking in Generalized Anxiety Disorder is evident across different versions of the Iowa Gambling Task. Journal of Behavior Therapy and Experimental Psychiatry, 41(2), 165171. 
negotiating narrow doorways. Frontiers in Computational Neuroscience, 7, 190. https://doi.org/10.3389/fncom.2013.00190

781 Muralidharan, V., Yu, X., Cohen, M. X., \& Aron, A. R. (2019). Preparing to stop action increases beta band power in contralateral sensorimotor cortex. Journal of Cognitive Neuroscience, 31(5), 657-668.

Must, A., Horvath, S., Nemeth, V. L., \& Janka, Z. (2013). The Iowa GamblingTask in depression - what have we learned about sub-optimal decision-making strategies? Frontiers in Psychology, 4, 1-6. https://doi.org/10.3389/fpsyg.2013.00732

Napoli, A., \& Fum, D. (2010). Rewards and punishments in iterated decision making: An explanation for the frequency of the contingent event effect. 10th International

Newman, L. I., Polk, T. A., \& Preston, S. D. (2008). Revealing individual differences in the Iowa Gambling Task. 1067-1072.

Oberg, S. A., Christie, G. J., \& Tata, M. S. (2011). Problem gamblers exhibit reward hypersensitivity in medial frontal cortex during gambling. Neuropsychologia, 49(13), $3768-3775$.

Ojeda, A., Kreutz-Delgado, K., \& Mishra, J. (2021). Bridging M/EEG Source Imaging and Independent Component Analysis Frameworks Using Biologically Inspired Sparsity Priors. Neural Computation, 33, 1-31. https://doi.org/10.1162/NECO_A_01415 learning for EEG source imaging. Neuroimage, 174, 449-462.

Pascual-Marqui, R. D., Michel, C. M., \& Lehmann, D. (1994). Low resolution electromagnetic tomography: A new method for localizing electrical activity in the brain. International 
Journal of Psychophysiology, 18(1), 49-65. https://doi.org/10.1016/01678760(84)90014-X

804 Paulus, M. P., \& Frank, L. R. (2006). Anterior cingulate activity modulates nonlinear decision weight function of uncertain prospects. Neuroimage, 30(2), 668-677.

Pfurtscheller, G. (1999). EEG event—Related desynchronization (ERD) and event-Releated synchronization (ERS). Electroencephalography: Basic Principles, Clinical Applications

Picazio, S., Veniero, D., Ponzo, V., Caltagirone, C., Gross, J., Thut, G., \& Koch, G. (2014).

Pizzagalli, D. A., Sherwood, R. J., Henriques, J. B., \& Davidson, R. J. (2005). Frontal brain Inhibition. Current Biology, 24(24), 2940-2945.

Pizzagalli, D. A., Webb, C. A., Dillon, D. G., Tenke, C. E., Kayser, J., Goer, F., Fava, M., asymmetry and reward responsiveness: A source-localization study. Psychological McGrath, P., Weissman, M., Parsey, R., Adams, P., Trombello, J., Cooper, C., Deldin, P., and risk in human subcortical structures. Neuron, 51(3), 381-390. https://doi.org/10.1016/j.neuron.2006.06.024 
825 Roca, M., Torralva, T., López, P., Cetkovich, M., Clark, L., \& Manes, F. (2008). Executive Functions in Pathologic Gamblers Selected in an Ecologic Setting. Cognitive and Behavioral Neurology, 21(1), 1-4. https://doi.org/10.1097/WNN.0b013e3181684358

828 Roelfsema, P. R., \& Ooyen, A. van. (2005). Attention-gated reinforcement learning of internal representations for classification. Neural Computation, 17(10), 2176-2214.

830 Schultz, W. (2000). Multiple reward signals in the brain. Nature Reviews. Neuroscience, 1(3), 199.

Silvetti, M., Wiersema, J. R., Sonuga-Barke, E., \& Verguts, T. (2013). Deficient reinforcement learning in medial frontal cortex as a model of dopamine-related motivational deficits in ADHD. Neural Networks, 46, 199-209.

Sims, C. R., Neth, H., Jacobs, R. A., \& Gray, W. D. (2013). Melioration as rational choice: Sequential decision making in uncertain environments. Psychological Review, 120(1),

838 Singh, V., \& Khan, A. (2012). Decision making in the reward and punishment variants of the Iowa gambling task: Evidence of "foresight" or "framing"? Frontiers in Neuroscience, 6,

841 Spitzer, R. L., Kroenke, K., Williams, J. B. W., \& Loewe, B. (2006). A Brief Measure for Assessing Generalized Anxiety Disorder: The GAD-7. Archives of Internal Medicine,

844 Stocco, A., Fum, D., \& Napoli, A. (2009). Dissociable processes underlying decisions in the Iowa Gambling Task: A new integrative framework. Behavioral and Brain Functions, 5(1), 1-12. 
847 Stopczynski, A., Stahlhut, C., Larsen, J. E., Petersen, M. K., \& Hansen, L. K. (2014). The smartphone brain scanner: A portable real-time neuroimaging system. PloS One, 9(2), e86733.

850 Sutton, R. S., \& Barto, A. G. (1998). Reinforcement Learning: An Introduction. Adaptive Computations and Machine Learning. MIT Press/Bradford.

852 Tobler, P. N., Christopoulos, G. I., O’Doherty, J. P., Dolan, R. J., \& Schultz, W. (2009). Riskdependent reward value signal in human prefrontal cortex. Proceedings of the National

Tops, M., \& Boksem, M. A. S. (2011). A potential role of the inferior frontal gyrus and anterior insula in cognitive control, brain rhythms, and event-related potentials. Front. Psychol. 2

Weller, J. A., Levin, I. P., \& Bechara, A. (2010). Do individual differences in Iowa Gambling Task performance predict adaptive decision making for risky gains and losses? Journal of

861 Wilson, R. C., \& Collins, A. G. (2019). Ten simple rules for the computational modeling of behavioral data. ELife, 8, e49547. https://doi.org/10.7554/eLife.49547

863 Woodrow, A., Sparks, S., Bobrovskaia, V., Paterson, C., Murphy, P., \& Hutton, P. (2019).

864 Decision-making ability in psychosis: A systematic review and meta-analysis of the magnitude, specificity and correlates of impaired performance on the Iowa and

867 Yoshimura, S., Okamoto, Y., Onoda, K., Matsunaga, M., Ueda, K., Suzuki, S., \& ShigetoYamawaki. (2010). Rostral anterior cingulate cortex activity mediates the 
relationship between the depressive symptoms and the medial prefrontal cortex activity. Journal of Affective Disorders, 122(1), 76-85. https://doi.org/10.1016/j.jad.2009.06.017

871 Zavala, B., Jang, A., Trotta, M., Lungu, C. I., Brown, P., \& Zaghloul, K. A. (2018). Cognitive

872 control involves theta power within trials and beta power across trials in the prefrontal-

873 subthalamic network. Brain: A Journal of Neurology, 141(12), 3361-3376.

874 https://doi.org/10.1093/brain/awy266

875 Ziegler, S., Pedersen, M. L., Mowinckel, A. M., \& Biele, G. (2016). Modelling ADHD: A

876 review of ADHD theories through their predictions for computational models of

877 decision-making and reinforcement learning. Neuroscience \& Biobehavioral Reviews, 71,

878 633-656. https://doi.org/10.1016/j.neubiorev.2016.09.002

879 